ISSN 0103-5150

Fisioter. Mov., Curitiba, v. 25, n. 1, p. 117-125, jan./mar. 2012 Licenciado sob uma Licença Creative Commons

\title{
Efeitos dos tratamentos fisioterapêutico e odontológico em pacientes com disfunção temporomandibular
}

\author{
Effects of treatments in dental physiotherapists and \\ patients with temporomandibular disorders
}

\section{Flavia Torres ${ }^{[a]}$, Ludmila Guterres Campos ${ }^{[b]}$, Helena Fetter Fillipini ${ }^{[c]}$, Karen Loureiro Weigert ${ }^{[d]}$, Giovana Formolo Dalla Vecchia ${ }^{[e]}$}

[a] Graduada em Fisioterapia pela Universidade Luterana do Brasil (ULBRA), Cachoeira do Sul, RS - Brasil, e-mail: flaviaschmidtorres@hotmail.com

[b] Mestre em Ciencias Aplicadas a la Atividad Física y Deporte pela Universidade de Córdoba, Espanha, professora do curso de Fisioterapia da Universidade Luterana do Brasil (ULBRA), Cachoeira do Sul, RS - Brasil, e-mail: benefisio@terra.com.br

[c] Mestre em Endodontia pela Universidade Luterana do Brasil (ULBRA), professora do curso de Odontologia da Universidade Luterana do Brasil (ULBRA), Cachoeira do Sul, RS - Brasil, e-mail: endofil@brturbo.com.br

[d] Mestre em Periodontia, pela Universidade Luterana do Brasil (ULBRA), professora do curso de Odontologia da Universidade Luterana do Brasil (ULBRA), Cachoeira do Sul, RS - Brasil, e-mail gvecchia@hotmail.com

[e] Doutora em Estomatologia Clínica pela Pontifícia Universidade Católica do Rio Grande do Sul, professora do curso de Odontologia da Universidade Luterana do Brasil (ULBRA), Cachoeira do Sul, RS - Brasil, e-mail: kweigert@piq.com.br

\section{Resumo}

Objetivos: Verificar os efeitos dos tratamentos odontológico e fisioterapêutico na redução da dor em pacientes com disfunção temporomandibular (DTM). Materiais e métodos: A amostra foi composta por dez pacientes (idade média de 34,3 anos) com DTM. Foram utilizados como instrumentos de coleta de dados o índice clínico de severidade para DTM, a escala visual analógica de dor (EVA) e a versão brasileira do Questionário McGill de Dor (Br-MPQ). Cinco pacientes realizaram o tratamento fisioterapêutico, com dez sessões (três semanas) de aplicações de estimulação elétrica nervosa transcutânea (Tens), ultrassom, massoterapia e alongamento; e cinco pacientes realizaram o tratamento odontológico com procedimentos farmacológicos e a confecção de uma placa miorrelaxante, usada por três semanas. Resultados: Comparando-se as médias dos resultados diários pré e pós-tratamento fisioterapêutico, houve redução significativa ( $<<0,05$ ) do quadro álgico em 69,3\%. Analisando-se a dor inicial e final, observou-se redução de 96,5\% no tratamento fisioterapêutico $(\mathrm{p}<0,05)$ e de 30,9\% no tratamento odontológico $(\mathrm{p}>0,05)$. No Br-MPQ a 
média de redução no tratamento fisioterapêutico, por categorias, foi de 92,5\% na categoria sensorial, 80\% na afetiva, $80 \%$ na avaliação subjetiva e $92 \%$ na mista; e no tratamento odontológico foi de $40,1 \%$ na categoria sensorial, $51,9 \%$ na afetiva, $34 \%$ na avaliação subjetiva e $55,9 \%$ na mista. Quanto à intensidade, no tratamento fisioterapêutico houve diminuição de 83,6\% (p<0,05) e no odontológico foi de 43,75 (p > 0,05). Conclusões: Ambos os tratamentos demonstraram ser positivos para o alívio da sintomatologia dolorosa em pacientes com DTM, sendo a fisioterapia, neste caso, um tratamento indicado, com melhora significativa da dor a curto prazo

Palavras-chave: Articulação temporomandibular. Fisioterapia. Odontologia.

\section{Abstract}

Objectives: To investigate the effects of dental treatments and physical therapy in reducing pain in patients with temporomandibular disorder (TMD). Materials and methods: The sample consisted of ten patients (mean age 34.3 years) with TMD. Used as instruments for data collection index clinical severity for TMD, visual analog scale for pain (VAS) and the Brazilian version of the McGill Pain Questionnaire (Br-MPQ). Five patients underwent physical therapy, with 10 sessions (three weeks) application of transcutaneous electrical nerve stimulation (Tens), ultrasound, massage and stretching, and five patients underwent dental treatment with pharmacological procedures and the making of a plate miorelaxante, used for three weeks. Results: Comparing the mean daily results before and after physical therapy, decreased significantly $(p<0.05)$ of pain in $69.3 \%$. Analyzing the initial and final pain, there was a decrease of $96.5 \%$ in physical therapy $(p<0.05)$ and $30.9 \%$ in dental treatment $(p>0.05)$. In the Br-MPQ the mean reduction in physical therapy, by category, was $92.5 \%$ in the sensory category, $80 \%$ in affective, $80 \%$ in the subjective evaluation and $92 \%$ in mixed and dental treatment was $40.1 \%$ in the sensory category, $51.9 \%$ in the affective, $34 \%$ in the subjective evaluation and $55.9 \%$ in mixed. As for intensity, physical therapy decreased from 83.6\% ( $p<0.05)$ and in dentistry was $43.75(p>0.05)$. Conclusions: Both treatments proved to be positive for the relief of painful symptoms in patients with TMD physiotherapy in this case a recommended treatment, with significant improvement in short-term pain.

Keywords: Temporomandibular joint. Physiotherapy. Dentistry.

\section{Introdução}

Segundo a American Association of Orofacial Pain (1), a disfunção temporomandibular (DTM) é um termo designado a um subgrupo das dores orofaciais, cujos sinais e sintomas incluem dor ou desconforto na articulação temporomandibular (ATM); nos ouvidos; músculos mastigatórios e cervicais, de um ou ambos os lados; estalidos; crepitação; amplitude de movimento mandibular limitada; desvios; e dificuldade de mastigação.

Conforme a literatura, os sinais e sintomas clássicos dessas disfunções compreendem dor de ouvido, dor facial, cefaleia, neuralgias, zumbidos, travamentos, dores musculares e articulares, limitações e desvios da trajetória mandibular, ruídos articulares durante a abertura e o fechamento bucal, dores de cabeça, nuca e pescoço $(2,3)$. Esses sinais e sintomas não são necessariamente estados de doença ou de necessidade de tratamento, porém, estima-se que $5 \%$ a $20 \%$ dos indivíduos precisarão ser tratados de alguma forma $(4,5)$.

A dor é um dos sintomas mais comuns na DTM, podendo haver variação com relação ao seu grau. Podem aparecer fadiga muscular e espasmos, derivados da hiperatividade muscular, que causam incoordenação ou disfunções dos músculos mastigatórios, dos músculos da cabeça e pescoço $(6,7)$.

Segundo a Sociedade Brasileira para o Estudo da Dor (8), a escala visual analógica (EVA) é utilizada para avaliar a dor em diversas patologias, incluindo as DTMs. Ela permite mensurar subjetivamente desde a ausência de dor até o mais alto grau de dor. Além da EVA, foi desenvolvido um instrumento validado para avaliação da dor, que consiste na versão brasileira do Questionário McGill de Dor (Br-MPQ), que além de mensurar as diferentes qualidades da dor, fornece medidas quantitativas, que podem ser 
tratadas estatisticamente e tem índices de validade e confiabilidade estabelecidos, com poder discriminativo entre os diversos componentes da dor $(9,10)$.

0 índice anamnésico (IA) proposto por Fonseca et al. (11) é um dos instrumentos disponíveis na língua portuguesa para a caracterização dos sintomas de DTM e foi desenvolvido para classificar os pacientes de acordo com a severidade desses sintomas. Recomenda-se que serviços de saúde pública e de triagem utilizem o IA para rastreamento dos portadores de DTM em razão de sua simplicidade, rapidez e baixo custo, atuando como uma ferramenta para rastreamento preliminar da DTM $(12,13)$.

Em razão da etiologia multifatorial, as DTMs exigem uma abordagem terapêutica interdisciplinar, mediante uma equipe formada por vários especialistas (cirurgião-dentista, fisioterapeuta, psicólogo e fonoaudiólogo) ou pelo menos uma estreita colaboração entre eles, uma vez que o tratamento bem-sucedido das DTMs deve envolver uma abordagem transdisciplinar e individualizada para cada paciente $(14,15)$.

Os pacientes reagem de formas diferentes às diversas terapias, e o profissional deve adequar o tratamento ao paciente para obter os melhores resultados possíveis. Portanto, apesar de os dentistas serem os profissionais envolvidos primeiramente no tratamento das DTMs, frequentemente os pacientes apresentam problemas posturais, tornando necessária a ação conjunta com o fisioterapeuta $(16,17)$.

0 tratamento fisioterapêutico baseia-se, de uma forma geral, em exercícios, massagens, alongamentos, terapia de liberação posicional (TLP), estimulação elétrica nervosa transcutânea (Tens), ultrassom e laser. Nos casos em que a DTM está relacionada com alterações posturais, a fisioterapia mostra-se efetiva nos objetivos de evitar a cirurgia, reposicionar a mandíbula, minimizar a dor muscular, melhorar a amplitude de movimento, melhorar a postura, reduzir a inflamação, reduzir a carga na ATM e fortalecer o sistema musculoesquelético (18-21).

No tratamento odontológico, é feita inicialmente uma avaliação e, de acordo com o quadro clínico, poderá ser realizado o aconselhamento do paciente, a prescrição de medicamentos analgésicos, anti-inflamatórios, relaxantes musculares ou reposição vitamínica. Além disso, poderão ser confeccionadas placas oclusais para o tratamento da DTM $(22,23)$.

Um claro senso de diagnóstico diferencial, para sentir o momento correto de indicar o paciente para outro profissional, permite a responsabilidade compartilhada na avaliação e no tratamento de cada situação clínica, além da aplicação combinada de equipamentos e técnicas especiais $(24,25)$.

Felizmente, hoje podemos observar uma crescente integração das diversas áreas da saúde, proporcionando ao paciente a cura efetiva, já que o ser humano é um "todo" em funcionamento, e não partes isoladas em ação. Um exemplo disso deve ser a atuação da fisioterapia e da odontologia no tratamento de pacientes com DTM. Entretanto, na população estudada essa integração ainda não é considerada comum entre os profissionais das referidas áreas; portanto, este estudo tem por objetivo verificar e analisar a atuação da fisioterapia e da odontologia no tratamento das DTMs (26).

\section{Materiais e métodos}

0 presente estudo caracterizou-se como um estudo do tipo ensaio clínico, pois comparou os efeitos pré e pós-intervenção odontológica e fisioterapêutica na redução da dor em pacientes com DTM.

Inicialmente, o projeto de pesquisa foi submetido ao Comitê de Ética em Pesquisa e à Comissão Nacional de Ética em Pesquisa. Após a sua aprovação ocorreu a seleção dos indivíduos, mediante a lista de espera da Clínica Escola de Odontologia da Ulbra, Câmpus Cachoeira do Sul, no período entre agosto e outubro de 2009, indivíduos esses que se enquadraram nos critérios de inclusão e exclusão. Eles assinaram um Termo de Consentimento Livre e Esclarecido e foram encaminhados para a clínica-escola do curso de Odontologia e para o laboratório do curso de Fisioterapia, onde foram realizados os tratamentos conforme o grupo a que pertenciam.

A amostra do estudo contou com dez sujeitos, divididos aleatoriamente em dois grupos, sendo o primeiro grupo composto por cinco pacientes tratados apenas com o tratamento fisioterapêutico; e o segundo composto também por cinco pacientes tratados apenas com o tratamento odontológico.

Participaram deste estudo pacientes que apresentavam dor na região da ATM, musculatura mastigatória e nas estruturas adjacentes, não sendo uma disfunção de origem articular. Tais pacientes não poderiam ter realizado nenhum tipo de tratamento para essa disfunção há pelo menos um mês, nem possuir história de lesões traumáticas ou tratamento cirúrgico na região orofacial ou ser portador de 
doenças sistêmicas, degenerativas e/ou neoplásicas. Todos deveriam ter disponibilidade de tempo para comparecer às dez sessões de fisioterapia e às consultas odontológicas necessárias.

Para se chegar a um diagnóstico de DTM a partir da anamnese utilizou-se, para todos os pacientes, uma ficha com dados pessoais e o IA, além do diagnóstico feito por dentistas da clínica-escola do curso de Odontologia. Utilizou-se também o Br-MPQ e a EVA para medir a intensidade da sintomatologia dolorosa.

No tratamento fisioterapêutico foram realizadas dez sessões, com duração de 50 minutos, durante um período de três semanas, com aplicação de Tens (pulso quadrado bifásico simétrico, $100 \mathrm{~Hz}, 80 \mu \mathrm{s}$, intensidade no limiar agradável individual de cada paciente) por 25 minutos, ultrassom (pulsado: $50 \%$, frequência de $1 \mathrm{MHz}$, potência $4 \mathrm{~W}$ ) por cinco minutos em cada articulação, e por último massagem na face com desativação de pontos-gatilhos de dor e manobras de alongamento e relaxamento cervical. No primeiro e último atendimentos foram realizadas as avaliações da dor segundo o Br-MPQ, EVA (em todas as sessões) e do IA.

No tratamento odontológico foram realizadas consultas em que, conforme o quadro clínico do paciente, foram feitas prescrições de medicamentos, aconselhamento e a confecção de placas miorrelaxantes, que no momento da instalação eram ajustadas em todos os pacientes, sendo que, antes e após três semanas de uso, foram aplicados o Br-MPQ a escala analógica visual (EAV) e o IA.

Os dados foram analisados segundo os procedimentos estatísticos descritivos (valores percentuais) e interferências (teste $t$ para as duas amostras em par para médias). 0 nível de significância utilizado foi de 0,05 .

\section{Resultados}

Entre o total de participantes que receberam tratamento fisioterapêutico ( $\mathrm{n}=05$ ), 60\% foram classificados como portadores de DTM severa, $40 \%$ como DTM moderada e $0 \%$ como DTM leve antes do tratamento. Depois do tratamento, nenhum $(0 \%)$ dos participantes foi classificado como portador de DTM severa, $40 \%$ como DTM moderada e $60 \%$ como DTM leve.

No grupo que recebeu tratamento odontológico $(\mathrm{n}=05), 80 \%$ dos participantes foram classificados como portadores de DTM severa, $20 \%$ como DTM moderada, $0 \%$ como DTM leve e $0 \%$ como não portador de DTM antes do tratamento. Depois do tratamento, $60 \%$ foram classificados como portadores de DTM severa, nenhum (0\%) como DTM moderada, $20 \%$ como DTM leve e $20 \%$ como não portador de DTM.

Na Tabela 1 pode-se perceber que todos os pacientes que realizaram o tratamento fisioterapêutico apresentaram melhora no quadro doloroso, segundo os valores da EVA, sendo essa melhora significante estatisticamente $(\mathrm{p}=0,0445)$ quando comparadas as médias pré e pós-intervenção fisioterapêutica.

O Gráfico 1 apresenta a comparação de antes e depois do tratamento fisioterapêutico, em cada paciente, no que se refere ao índice de dor, sendo que o paciente $A$ apresentou na avaliação inicial um valor de 6,7 na EVA, e esse valor caiu para 0 na avaliação final, diminuindo seu quadro doloroso em $100 \%$. 0 paciente $B$, na avaliação inicial, apresentou o valor de 3,1, passando para 0 na avaliação final, diminuindo a dor em $100 \%$. 0 paciente $C$ apresentou o valor inicial de 0,3 e o final de 0 diminuindo a dor em $100 \%$. 0 paciente D tinha como valor inicial 2,4 e diminuiu para 0,6 , diminuindo a dor em $75 \%$. 0 paciente $E$ diminuiu seu valor de 4,8 para 0 , diminuindo sua dor em $100 \%$.

A Tabela 2 mostra que todos os pacientes que realizaram o tratamento odontológico apresentaram melhora no quadro doloroso, segundo os valores da EVA, entretanto essa melhora não foi significante estatisticamente $(\mathrm{p}=0.3443)$ quando comparados os índices de dor pré e pós-intervenção odontológica.

Tabela 1 - Análise estatística comparativa do índice de dor entre o Br-MPQ e a EVA antes e depois do tratamento fisioterapêutico

\begin{tabular}{lccc}
\hline 1. Br-MPQ & $\overline{\mathbf{x}}$ & $\mathbf{s}$ & $\mathbf{p}$ \\
\hline Antes & 13,4 & 6,5 & 0.0151 \\
Depois & 2,2 & 5 & \\
\hline 2. Escala analógica visual & $\overline{\mathbf{x}}$ & $\mathbf{s}$ & $\mathbf{p}$ \\
\hline Antes & 2,31 & 1,35 & 0.0445 \\
Depois & 0,71 & 0,66 & \\
\hline
\end{tabular}

Fonte: Dados da pesquisa. 


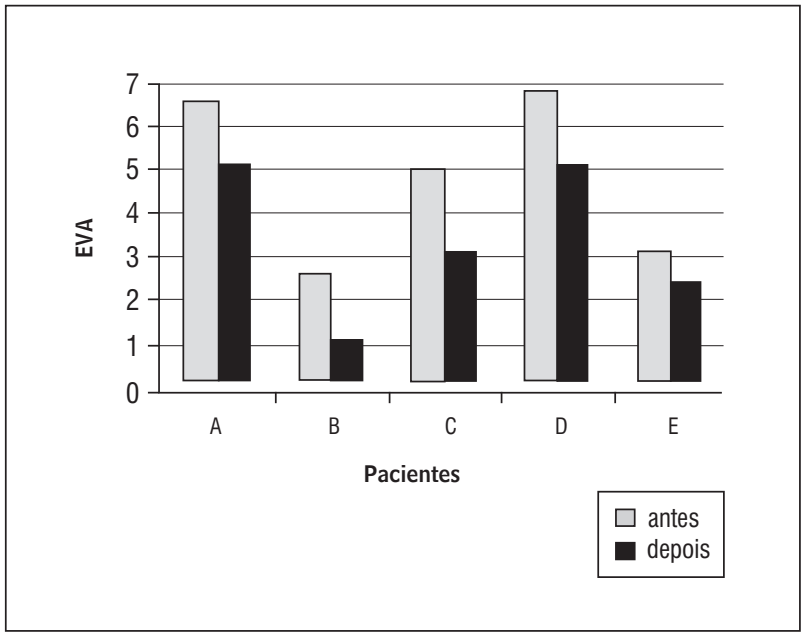

Gráfico 1 - Evolução do grupo submetido ao tratamento fisioterapêutico, quanto ao nível de dor antes e depois do tratamento

Fonte: Dados da pesquisa.

Tabela 2 - Análise estatística comparativa do índice de dor entre o Br-MPQ e a EAV antes e depois do tratamento odontológico

\begin{tabular}{lccc}
\hline 1. Br-MPQ & $\overline{\mathbf{x}}$ & $\mathbf{s}$ & $\mathbf{p}$ \\
\hline Antes & 25,6 & 14 & \\
Depois & 14,4 & 12 & 0.2048 \\
\hline 2. Escala analógica visual & $\overline{\mathbf{x}}$ & $\mathbf{s}$ & $\mathbf{p}$ \\
\hline Antes & 4,72 & 1,9 & \\
Depois & 3,46 & 2 & \\
\hline
\end{tabular}

Fonte: Dados da pesquisa.

O Gráfico 2 apresenta a comparação de antes e depois do tratamento odontológico, em cada paciente, no que se refere ao índice de dor, sendo que o paciente $\mathrm{F}$ apresentou na avaliação inicial um valor de 6,5 na EVA, e esse valor caiu para 5 na avaliação final, diminuindo seu quadro doloroso em $23 \%$. 0 paciente $G$, na avaliação inicial, apresentou o valor de 2,5, passando para 1,0 na avaliação final, diminuindo a dor em $60 \%$. 0 paciente $\mathrm{H}$ apresentou o valor inicial de 4,9 e o final de 3,0, diminuindo a dor em $38,8 \%$. 0 paciente I tinha como valor inicial 6,7 e diminuiu para 6, diminuindo a dor em 10,4\%. 0 paciente J diminuiu seu valor de 3 para 2,3, diminuindo sua dor em $23,3 \%$.

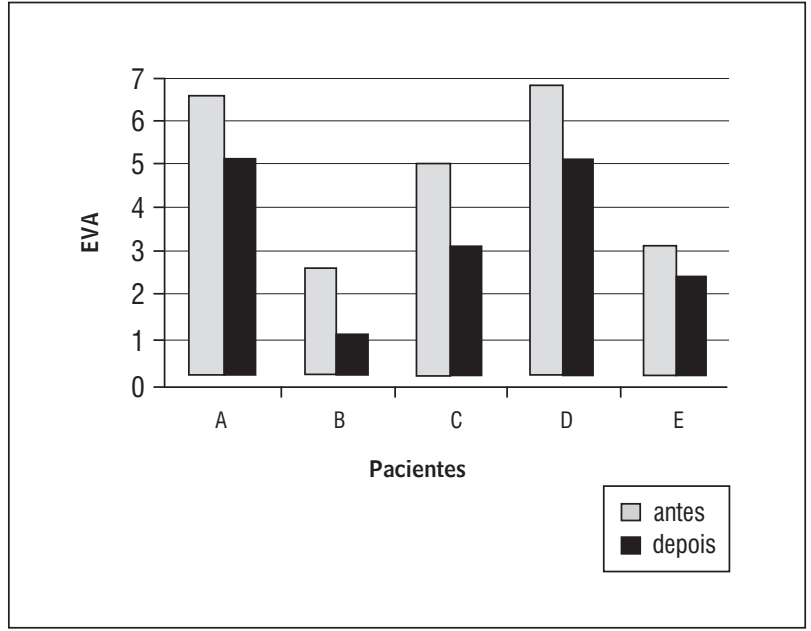

Gráfico 2 - Evolução do grupo submetido ao tratamento odontológico, quanto ao nível de dor antes e depois do tratamento

Fonte: Dados da pesquisa.

As categorias do Br-MPQ mostraram diminuições nos valores encontrados pré e pós-tratamento, sendo que para o grupo que recebeu fisioterapia apenas um paciente não zerou sua dor após o tratamento; portanto, para esse grupo houve diminuição média de 92,5\% na categoria sensorial, $80 \%$ na afetiva, $80 \%$ na avaliação subjetiva e $92 \%$ na mista. No grupo que recebeu tratamento odontológico, nenhum paciente reduziu totalmente seu índice de dor, sendo que esse grupo mostrou redução média de $40,1 \%$ na categoria sensorial, 51,9\% na afetiva, $34 \%$ na avaliação subjetiva e 55,9\% na mista, como mostra o Gráfico 3. Quanto à intensidade de dor, no grupo que recebeu fisioterapia houve diminuição de $83,6 \%(p=0,0151)$ e no grupo que recebeu tratamento odontológico essa diminuição foi de $43,75 \%(p=0,2048)$.

\section{Discussão}

Participaram do estudo dez pacientes, sendo $10 \%$ do gênero masculino e $90 \%$ do gênero feminino, concordando com a literatura que refere que as mulheres possuem mais sintomas de DTM que os homens (27-30).

A média de idade encontrada foi de 34,3 anos, semelhante aos dados registrados por outros pesquisadores (31-33), e ainda concorda com as diretrizes apontadas pela Academia Americana de Dor 


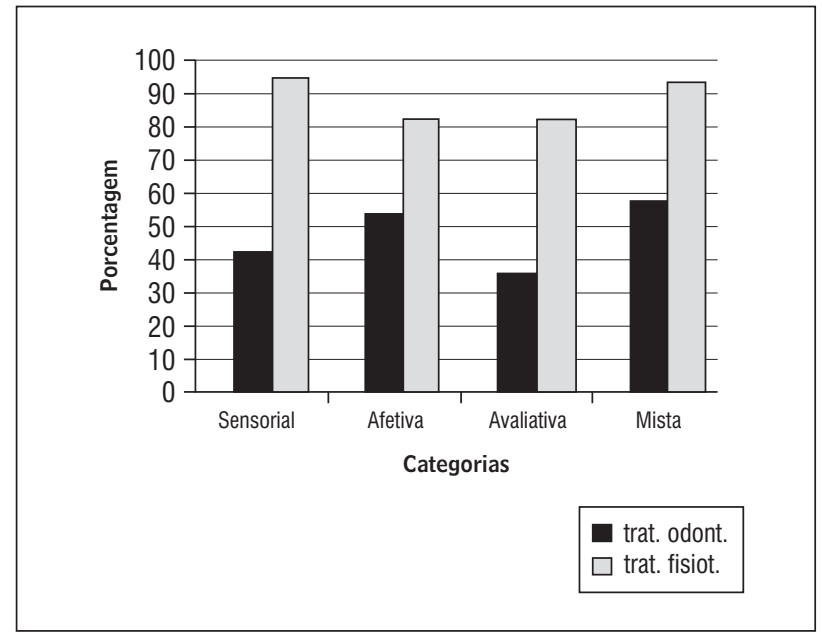

Gráfico 3 - Média de redução dos dois grupos na pontuação no Br-MPQ por dimensões

Fonte: Dados da pesquisa.

Orofacial, que preconizou a prevalência entre a segunda e a quarta décadas de vida, além de outros autores que referem existir maior predisposição do gênero feminino em desenvolver a DTM na idade produtiva $(34,35)$.

Para o tratamento odontológico, Okeson (36) refere que o uso das placas oclusais compreende uma modalidade de tratamento com alto índice de sucesso na diminuição dos sintomas de DTMs miogênicas (37). Entretanto, embora as placas oclusais tenham demonstrado sucesso em grande número de estudos, Portero et al. (38) ressaltam que elas não devem ser usadas como única modalidade de tratamento, mas sim como parte desse ou até mesmo como coadjuvante de outras terapias, como a medicamentosa ou a fisioterapêutica, sendo necessário que se descubra a etiologia da DTM para que se obtenha sucesso a longo prazo no tratamento.

Portanto, o presente estudo concorda que as placas oclusais só serão efetivas se o paciente as estiver usando corretamente $(38,39)$, sendo que não existe uma placa oclusal que seja útil para tratar todas as DTMs, existindo algumas que não respondem à terapia por placas, sendo então necessária a instituição de outras modalidades de tratamento (40), como o fisioterapêutico.

Ainda no que se refere à atuação da odontologia nas DTMs, na literatura percebe-se o amplo uso das placas oclusais e a mínima utilização de aconselhamento/farmacoterapia como modalidades terapêuticas (41). 0 mesmo foi encontrado no grupo que recebeu tratamento odontológico, pois apenas dois pacientes foram tratados com farmacologia aliada à placa oclusal. Entretanto, Alencar, Ribeiro e Rodrigues (42), ao avaliarem 258 pacientes tratados com placas oclusais ou farmacoterapia/aconselhamento, verificaram que $24 \%$ tiveram indicação para o uso de placa oclusal com aconselhamento e $76 \%$ receberam apenas o aconselhamento e/ou farmacoterapia, contrariando a literatura.

Macedo (43), ao realizar uma revisão de literatura para verificar a efetividade das placas oclusais, percebeu que não há evidência suficiente na literatura para se afirmar que as placas oclusais são efetivas para o tratamento do bruxismo do sono, mas pode ser que haja alguns benefícios com relação ao desgaste dentário, como pode ter sido o caso do grupo que recebeu tratamento odontológico, embora esse grupo também tenha melhorado quanto à sintomatologia dolorosa.

Silva et al. (44), ao analisarem eletromiograficamente o efeito imediato da placa oclusal no equilíbrio muscular em dez pacientes com DTM, concluiram que as placas oclusais foram eficazes na manutenção do equilíbrio muscular dos portadores de DTM após 70 dias de uso. Portanto, o curto período de avaliação do presente estudo pode ter influenciado nos resultados obtidos, uma vez que não foram feitos os ajustes necessários para instituir maior equilíbrio musculoesquelético.

Assim como no grupo que recebeu tratamento odontológico neste estudo, Sima \& Gil (45) compararam a severidade dos sinais e sintomas das DTMs em 25 pacientes com idade média de 30,7 anos, submetidos a tratamento por meio da utilização de placa oclusal (uso noturno), acompanhados e avaliados por um período de 30 dias após a sua instalação, concluindo que os pacientes apresentaram prevalência significantemente maior no grau de severidade antes do uso da placa oclusal.

El Fatih, Ibrahim e El Laithi (46), utilizando protocolo fisioterapêutico semelhante ao do grupo que recebeu fisioterapia neste estudo, verificaram a eficácia do tratamento fisioterapêutico em 57 pacientes com DTM, divididos em dois grupos. Um grupo recebeu aplicações de Tens e o outro grupo recebeu aplicações de ultrassom. Ao avaliar a dor com a EVA, verificou-se diminuição significativa em ambos os grupos, concordando com os resultados encontrados neste estudo. 
Concordando com o recurso utilizado no grupo que recebeu tratamento com fisioterapia, Rodrigues, Siriani e Bérzin (47) avaliaram o efeito da Tens sobre a dor de 19 indivíduos com DTM e 16 normais, verificando-se que nos portadores de DTM a Tens reduziu a dor, a atividade eletromiográfica da porção anterior do músculo temporal e aumentou a atividade dos músculos masseteres durante a contração máxima voluntária, concluindo que apenas uma aplicação da Tens é efetiva para reduzir a dor em pacientes com DTM.

Guerra (48), ao avaliar a eficácia do ultrassom no alívio da dor em 26 pacientes com DTM, sendo que $61,53 \%$ destes possuíam DTM severa e $38,46 \%$ DTM moderada, segundo o IA, verificou que, após o tratamento, $15,38 \%$ possuíam DTM severa, $34,61 \%$ DTM moderada, $46,15 \%$ DTM leve e 3,84\% foram classificados como não portadores de DTM, mostrando que o tratamento com ultrassom pulsado produz melhora significativa das queixas.

Biasotto (49) avaliou a eficácia da técnica de massoterapia em 60 indivíduos e verificou, por meio da EVA, diminuição significativa da dor e da atividade eletromiográfica no grupo tratado com massoterapia, confirmando a eficácia dessa técnica em portadores de DTM miogênica. Mais tarde, Eisensmith (50) investigou a eficácia da massagem terapêutica para minimizar a frequência e a intensidade dos sintomas de DTM, concluindo que técnicas de massagem podem melhorar a amplitude de movimento da mandíbula, aliviar a intensidade e reduzir a frequência da dor na ATM.

Para verificar o efeito do tratamento integrado entre fisioterapia e odontologia no quadro álgico de pacientes com DTM, Troian (51) utilizou duas abordagens de tratamento: uma por meio do atendimento odontológico e outra integrando o atendimento odontológico e fisioterapêutico, havendo, nessa última, diminuição mais evidente da intensidade de dor que nos pacientes que receberam somente o tratamento odontológico.

\section{Referências}

1. American Association of Orofacial Pain. [Acesso em: 25 ago 2009]. Disponível em: http://www.aaop.org/content.aspx?page_id=22\&club_id=508439\&module_ id=107325.
2. Pereira KNF, Andrade LLS, Costa MLG, Portal TF. Sinais e sintomas de pacientes com disfunção temporomandibular. Rev CEFAC. 2005;7(2):221-28.

3. Santos ECA, Bertoz FA, Pignatta LMB, Arantes FM. Avaliação clínica de sinais e sintomas da disfunção temporomandibular em crianças. Rev Dental Press Ortodon Ortop Facial. 2006;11(2):29-34.

4. Tosato JP, Gonzalez TO, Sampaio LMM, Corrêa JCF, Biasotto-Gonzalez A. Prevalência de sinais e sintomas de disfunção temporomandibular em mulheres com cervicalgia e lombalgia. Arq Med ABC. 2007; 32(2):20-2.

5. Moreno BGD, Maluf SA, Marques AP, Crivello O Jr. Avaliação clínica e da qualidade de vida de indivíduos com disfunção temporomandibular. Rev Bras Fisioter. 2009;13(3):210-4.

6. Maciel RN. Oclusão e ATM: procedimentos clínicos. São Paulo: Santos; 1998.

7. Oliveira AS, Bermudez CC, Souza RA, Souza CMF, Castro CES. Impacto da dor na vida de portadores de disfunção temporomandibular. J Appl Oral Sci. 2003;11(2):138-43.

8. Dixon JS, Bird HA. Reproducibility along a $10-\mathrm{cm}$ vertical visual analogue scale. Ann Rheum Dis. 1981; 40(1):87-9.

9. Pimenta CAM, Teixeira MJ. Questionário de dor McGill: proposta de adaptação para a língua portuguesa. Rev Esc Enf USP. 1996;30(3):473-83.

10. Melzack R. The McGill pain questionnaire. From description to measurement. Anesthes. 2005; 103(1):199-202.

11. Chaves TC, Oliveira AS, Grossi DB. Principais instrumentos para avaliação da disfunção temporomandibular, parte I: índices e questionários; uma contribuição para a prática clínica e de pesquisa. Fisioter Pesq. 2008;15(1):92-100.

12. Campos JADB, Gonçalves DAG, Camparis CM, Speciali JG. Confiabilidade de um formulário para diagnóstico da severidade da disfunção temporomandibular. Rev Bras Fisioter. 2009;13(1):38-43.

13. Chaves TC, Costa D, Grossi DB, Bertolli F. Avaliação anamnésica de sintomas de disfunção temporomandibular em crianças asmáticas. Fisioter Pesq. 2005;11(1):19-26. 
14. Pereira FJ Jr, Vieira AR, Prado R, Miasato JM. Visão geral das desordens temporomandibulares. RGO. 2004;52(2):117-21.

15. Granja DVA, Lima AP. Influência dos recursos fisioterapêuticos nas algias orofaciais. Rev. Fisioter. Brasil. 2003;4(6):394-401.

16. Marzola FT, Marques AP, Marzola C. Contribuição da fisioterapia para a odontologia nas disfunções da articulação temporomandibular. Rev Odonto Ciência. 2002;17(36):119-34.

17. Corrêa ECR, Bérzin F. Temporomandibular disorder and dysfunctional breathing. Braz J Oral Sci. 2004; 3(10):498-502.

18. Castro FM, Gomes RCV, Salomão JR, Abdon APV. A efetividade da terapia de liberação posicional (TLP) em pacientes portadores de disfunção temporomandibular. Ver Odont Univ Cidade São Paulo. 2006; 18(1):67-74.

19. Medlicott MS, Harris SR. A systematic review of the effectiveness of exercise, manual therapy, electrotherapy, relaxation training, and biofeedback in the management of temporomandibular disorder. Physical Therapy. 2006;86(7):955-73.

20. Maluf SA, Moreno BGD, Alfredo PP. Exercícios terapêuticos nas desordens temporomandibulares: uma revisão de literatura. Fisioter Pesq. 2008; 15(4): 408-15.

21. Barbosa GAS, Barbosa KVMS, Badaró CR Filho. Recursos fisioterápicos disponíveis para o tratamento das disfunções temporomandibulares. JBA. 2003; 3(11):257-62.

22. Alencar JRF, Becker A. Evaluation of different occlusal splints and counselling in the management of myofascial pain dysfunction. J Oral Rehabil. 2009; 36(2):79-85.

23. Oliveira W. Disfunções temporomandibulares. São Paulo: Artes Médicas; 2002.

24. Rossi AC, Resende MCRA, Araújo OP Jr, Garcia AR, Zuim PRJ, Marinho LVD. Fisioterapia no tratamento multidisciplinar da disfunção temporomandibular. Rev Odonto UNESP. 2008;37(Número Especial):190.

25. Melo GM. Disfunções temporomandibulares e dores orofaciais: uma visão interdisciplinar para o tratamento. Rev Dentíst. 2008;8(17):1-5.
26. Póli MS, Morosini MRM, Martinelli RCPM. Abordagem interdisciplinar na disfunção temporomandibular: relato de caso. Arq Ciênc Saúde Unipar. 2003; $7(2): 171-7$.

27. Moresca R, Urias D. Estudo epidemiológico dos ruídos da ATM em adultos jovens sul-brasileiros. JBA. 2001;1(2):121- 9.

28. Shibayama R, Garci AR, Zuim PRJ. Prevalência de desordem temporomandibular (DTM) em pacientes portadores de duplas próteses parciais removíveis e universitários. Rev Odont Araçatuba. 2004; 25(2):18-21.

29. Silveira AM, Feltrin PP, Zanetti RV, Mautoni MC. Prevalência de portadores de DTM em pacientes avaliados no setor de otorrinolaringologia. Rev Bras Otorrinolaringol. 2007;73(4):528-32.

30. Warren MP, Fried JL. Temporomandibular disorders and hormones in women. Cells Tissues Organs. 2001;169(3):187-92.

31. Lima FAL, Toscano CFS, Silva JM Filho. Perfil epidemiológico de sujeitos com disfunção temporomandibular tratados na faculdade de odontologia de Caruaru - Pernambuco. Fisioter Mov. 2007; 20(4): 101-8.

32. Santos TS, Moura AD, Piva MR, Rabello M, Carvalho LPM, Martins PRS Filho. Relação dos desgastes dentários com as disfunções temporomandibulares. Rev Cir Traumatol. Buco-Maxilo-fac. 2009;9(2):73-80.

33. Rodrigues D, Siriani AO, Bérzin F. Effect of conventional TENS on pain and electromyographic activity of masticatory muscles in TMD patients. Braz Oral Res. 2004;18(4):290-5.

34. Lisboa MV, Albuquerque SR, Ferreira JC, Jesus AA, Lisboa JAA. Prevalência de gênero e idade de pacientes com disfunção temporomandibular no COATFOUFBA. Rev Fac Odontol Univ Fed Bahia. 2003; 27:15-18.

35. Tosato JP, Caria PHF. Prevalência de DTM em diferentes faixas etárias. RGO. 2006;54(3):211-24.

36. Okeson JP. Tratamento das desordens temporomandibulares e oclusão. São Paulo: Artes Médicas; 2000.

37. Machado EM, Conde MC, Cássio Carvalho CV. O uso de placas oclusais como coadjuvante do tratamento periodontal. Rev Odont USP. 2007;19(2):201-8. 
38. Portero PP, Kern R, Kusma SZ, Grullón PG. Placas oclusais no tratamento da disfunção temporomandibular (DTM). Rev Gest \& Saúd. 2009;1(1):36-40.

39. Ré JP, Perez C, Darmouni L, Carlier JF, Orthlieb JD. The occlusal splint therapy. J Stomat Occ Med. 2009; 2:82-6.

40. Hamata MM, Zuim PRJ, Garcia AR. Comparative evaluation of the efficacy of occlusal splints fabricated in centric relation or maximum intercuspation in temporomandibular disorders patients. J Appl Oral Sci. 2009;17(1):32-8.

41. Milam A, Fava ELF, Lino HE, Milam JRT, Lino HL Jr. Levantamento de incidência de DTMS e análise da efetividade da placa de mordida plana como terapia. Semina. 2004;25:23-38.

42. Alencar FGP, Ribeiro C, Rodrigues M. Avaliação longitudinal de pacientes com disfunções têmporomandibulares tratados com placas oclusais, aconselhamento e farmacoterapia. Robrac. 2006;15(40):63-70.

43. Macedo CR. Placas oclusais para tratamento do bruxismo do sono: revisão sistemática Cochrane. [dissertação]. São Paulo: Universidade Federal de São Paulo; 2007.

44. Silva ASS, Uchoa ES, Nóbilo MAA, Fausto B. Avaliação eletromiográfica da influência da placa oclusal sobre o orbicular da boca em indivíduos portadores de próteses totais com disfunção temporomandibular e dor orofacial. Rev Odonto Ciênc. 2007;22(57):263-8.

45. Sima FT, Gil C. Estudo comparativo do grau de severidade das desordens craniomandibulares em pacientes edentados parciais antes e após a utilização de placas interoclusais. RPG. 2005;12(2):179-85.
46. El Fatih I A, Ibrahim AI, El Laithi A. Efficacy of physiotherapy and intraoral splint in the management of temporomandibular disorders. Saud Dent Journ. 2004;16(1):16-20.

47. Rodrigues D, Siriani AO, Bérzin F. Effect of conventional TENS on pain and electromyographic activity of masticatory muscles in TMD patients. Braz Oral Res. 2004;18(4):290-5.

48. Guerra LMC. Eficácia do ultra-som na terapia das Disfunções Temporomandibulares: avaliação clínica e eletromiográfica. [dissertação]. Pernambuco: Universidade Federal de Pernambuco; 2003.

49. Biasotto DA. Efeito da técnica Fisioterapeutica (massoterapia) em indivíduos portadores de Desordem Temporomandibular Miogênica: um estudo eletromiográfico. [dissertação]. Piracicaba: faculdade de Odontologia de piracicaba da Universidade Estadual de Campinas; 2002.

50. Eisensmith LP. Massage therapy decreases frequency and intensity of symptoms related to temporomandibular joint syndrome in one case study. J Bodywork Move Therap. 2007;11:223-30.

51. Troian MA. Tratamento interdisciplinar entre fisioterapia e odontologia na redução da dor em pacientes com disfunção do sistema craniocervicomandibular. Reabilit. 2005;7(26):29-39.

Recebido: 25/03/2011

Received: 03/25/2011

Aprovado: 21/07/2011

Approved: 07/21/2011 